\title{
(Non)standard oscillations at current facilities
}

\section{David Vanegas Forero*广}

Center for Neutrino Physics, Virginia Tech, Blacksburg, VA 24061, USA

E-mail: dvanegas@vt.edu

\begin{abstract}
Models that generate neutrino masses (and, in general, other SM extensions) might produce new neutrino interactions as a by-product. In the usual phenomenological framework, Non-Standard Neutrino Interactions (NSI) are modeled as a four fermion neutrino interaction with general couplings times the Fermi constant. We review the general formalism and the current limits on the NSI couplings. Finally, the NSI impact in the determination of the neutrino oscillation parameters is discussed in the context of the running facilities focusing on the determination of the reactor mixing angle and the Dirac CP phase.
\end{abstract}

Neutrino Oscillation Workshop

4 - 11 September, 2016

Otranto (Lecce, Italy)

\section{* Speaker.}

†I would like to thank the conveners of the 'Oscillation parameters: present' session for accepting my contribution. 
Neutrino oscillations are the main mechanism to describe neutrino flavor transitions observed at different facilities from several neutrino sources. In particular, neutrinos produced at man made sources like reactors and accelerators have been playing an important role towards the completion of the three neutrino oscillation framework. New facilities are also been planned to determine the two main unknowns in the neutrino sector: the determination of the mass ordering and to establish whether the charged-parity symmetry is also violated in the lepton sector, and also to improve the precision of the measured oscillation parameters. This 'precision era' also opens the possibility to probe scenarios beyond the standard oscillation framework. In particular non-standard neutrino interactions (NSI) provide an interesting framework that can be tested with neutrino oscillation data. NSI can be the low energy 'remnant' of different extensions of SM motivated for instance by neutrino masses. Interestingly, NSI were first proposed long time ago even before neutrino oscillations were discovered and to date they are still a viable beyond the SM scenario. In the following, we describe NSI effects at running neutrino oscillation facilities based on the works done in Refs. [1,2].

At low energies the neutrino charged current (CC) and neutral current (NC) interactions can be summarized in the following general form:

$$
\begin{aligned}
\mathscr{L}_{\mathrm{CC}-\mathrm{NSI}} & =\frac{G_{F}}{\sqrt{2}} \sum_{f, f^{\prime}} \varepsilon_{\alpha \beta}^{S D, f f^{\prime}}\left[\bar{v}_{\beta} \gamma^{\rho}\left(1-\gamma^{5}\right) \ell_{\alpha}\right]\left[\bar{f}^{\prime} \gamma_{\rho}\left(1 \pm \gamma^{5}\right) f\right], \\
\mathscr{L}_{\mathrm{NC}-\mathrm{NSI}} & =\frac{G_{F}}{\sqrt{2}} \sum_{f} \varepsilon_{\alpha \beta}^{f}\left[\bar{v}_{\beta} \gamma^{\rho}\left(1-\gamma^{5}\right) v_{\alpha}\right]\left[\bar{f} \gamma_{\rho}\left(1 \pm \gamma^{5}\right) f\right],
\end{aligned}
$$

where $G_{F}$ is the Fermi constant, $\varepsilon_{\alpha \beta}$ are dimensionless couplings whose magnitude sizes the NSI interaction. The $S, D$ superscript for NSI couplings in Eq. (1) differentiates NSI in the source from the detector while the $f f^{\prime}$ represents the fermion involved in the interaction. Even though other bilinear covariants combinations are possible, the SM-like $V \pm A$ structure in Eqs. (1) and (2) prevails for phenomenological reasons [3].

In Eqs. (1) and (2) we have done a convenient separation distinguishing the $\mathrm{CC}$ from the $\mathrm{NC}$ contribution. This is useful to evidence the NSI modifications to the neutrino phenomenology with only SM interactions. In the case on CC-like NSI in Eq. (1), in a CC weak process, there is a probability that a 'new' state $\left|v_{\gamma}\right\rangle$ be 'produced' with a different flavor of the lepton $l_{\alpha}$. This neutrino flavor transition can happen in the source or in the detector. To quantify this effect, the new flavor component is added to the antineutrino flavor state $\left|\bar{v}_{\alpha}^{s}\right\rangle=\left|\bar{v}_{\alpha}\right\rangle+\sum_{\gamma} \varepsilon_{\alpha \gamma}^{s *}\left|\bar{v}_{\gamma}\right\rangle^{1}$, where the standard flavor states are related to mass eigenstates by $\left|\bar{v}_{\alpha}\right\rangle=\sum_{k} U_{\alpha k}\left|\bar{v}_{k}\right\rangle$. The main consequence is that the basis defined by the new flavor states $\left|\bar{v}_{\alpha}^{s, d}\right\rangle$ is not longer complete, the usual flavor states $\left|\bar{v}_{\alpha}\right\rangle$ still define a complete basis.

Neutrinos propagating in a given medium interact with matter, SM fermions of the first family. In the case of SM interactions, the CC contribution from the $v_{e}-e$ interaction is encoded in the element $(1,1)$ of effective matter potential $V$. The NC contribution is the same for the three flavors (universal) and flavor diagonal contributing to $V_{i i}{ }^{2}$. Differently, the NC-like NSI in Eq. (2) opens

\footnotetext{
${ }^{1}$ Together with the ket $\left\langle\bar{v}_{\beta}^{d}\right|=\left\langle\bar{v}_{\beta}\right|+\sum_{\eta} \varepsilon_{\eta \beta}^{d *}\left\langle\bar{v}_{\eta}\right|$. To obtain the neutrino states one should conjugate the mixing matrices in the antineutrino definition.

${ }^{2}$ For this reason the SM NC contribution to $V_{i i}$ is generally rephased out by a redefinition of the neutrino estates.
} 
the possibility of having flavor changing NC processes therefore populating the off-diagonal elements of $V$. Also, the diagonal NSI couplings are different for different flavors thus non-universal.

In the case of neutrinos propagating in a medium of constant density, the general matter interaction Hamiltonian can then be written as:

$$
\mathscr{H}_{\mathrm{int}}=\tilde{V}\left(\begin{array}{ccc}
1+\varepsilon_{e e}^{m} & \varepsilon_{e \mu}^{m} & \varepsilon_{e \tau}^{m} \\
\left(\varepsilon_{e \mu}^{m}\right)^{*} & \varepsilon_{\mu \mu}^{m} & \varepsilon_{\mu \tau}^{m} \\
\left(\varepsilon_{e \tau}^{m}\right)^{*} & \left(\varepsilon_{\mu \tau}^{m}\right)^{*} & \varepsilon_{\tau \tau}^{m}
\end{array}\right),
$$

with $\tilde{V}=\sqrt{2} G_{F} N_{e}$, where $N_{e}$ is the electron density of the medium. Notice that the elements of the general potential are a combination of the fundamental couplings $\varepsilon_{\alpha \beta}^{m}=\sum_{f=e, u, d}\left\langle Y_{f} / Y_{e}\right\rangle \varepsilon_{\alpha \beta}^{f}=$ $\varepsilon_{\alpha \beta}^{e}+Y_{u} \varepsilon_{\alpha \beta}^{u}+Y_{d} \varepsilon_{\alpha \beta}^{d}$ where $Y_{f}$ is the $f$ relative abundance in the medium. The final step is to calculate (anti)neutrino transition probabilities in the presence of NSI $P_{\bar{v}_{\alpha}^{s} \rightarrow \bar{v}_{\beta}^{d}}=\left|\left\langle\bar{v}_{\beta}^{d}\left|\exp \left(-i \mathscr{H}_{\text {int }} L\right)\right| \bar{v}_{\alpha}^{s}\right\rangle\right|^{2}$ with the ultimate goal of obtaining the numbers of events in neutrino detectors.

\section{CC-like NSI signatures}

Reactor neutrino experiments, with $\sim 1 \mathrm{~km}$ baseline, have successfully measured the reactor mixing angle, and in the case of Daya Bay and RENO, this have been possible thanks to the reduction of systematical errors by the use of more than one detector and also due to the large number of events that are produced. At $1 \mathrm{~km}$ baseline, neutrino-matter interactions are negligible, and therefore, this kind of experiments are mainly sensitive to CC-like NSI. In this section we show that Daya Bay precise data is also powerful constraining NSI.

Before defining a specific setup, it is relevant to make a comment on previous NSI bounds. The relevant couplings are of the form $\varepsilon_{\alpha \beta}^{u d}$ and their bounds can be extracted from different weak processes: $V^{u d}$ determination (kaon versus beta decays), universality test, and from the nonobservation of flavor change in NOMAD. Limits, using these processes, were calculated using the one parameter at the time scheme with several assumptions in Ref. [4]. The main result is that, except for the $\varepsilon_{\tau \tau}^{u d}$, previous bounds on the NSI couplings are of the order of $\sim 0.01$.

Assuming the detection and production couplings are related by a 'complex conjugate' operation $^{3} \varepsilon_{e \alpha}^{s}=\varepsilon_{\alpha e}^{d *} \equiv \varepsilon_{\alpha}=\left|\varepsilon_{\alpha}\right| \mathrm{e}^{i \phi_{\alpha}}$ (also removing $f=u, f^{\prime}=d$ from the superscript), which is consistent with the form $\bar{v}_{e}$ are produced (via $\beta$-decay) and detected (via inverse $\beta$-decay), the following expression for the effective $\bar{v}_{e}$ disappearance probability is obtained: $P_{\bar{v}_{e}^{s} \rightarrow \bar{v}_{e}^{d}} \approx$ $1+4\left|\varepsilon_{e}\right| \cos \phi_{e}-4 \sin ^{2} \theta_{13}^{\text {eff. }} \sin ^{2} \Delta_{31}$, where $\sin ^{2} \theta_{13}^{\text {eff. }}=f\left(\theta_{i j} ; \varepsilon_{\mu(\tau)}\right)$ and $\Delta_{31} \equiv\left(\Delta m_{31}^{2}\right) L /(4 E)$. Notice that reactor experiments are sensitive to the $\varepsilon_{e \beta}^{u d}$ couplings. The main consequences of this expression are the possibility of flavor transition at $L=0$ (zero distance effect) and the redefinition of the reactor mixing angle. After performing a total rate analysis of the Daya Bay data, accumulated in 621 days, we improved the constraint on $\varepsilon_{e e}^{u d}$ respect to one in Ref. [4]. However, the main result of our paper is that this constraint is subjected to the treatment of the error on the total normalization of the reactor fluxes ${ }^{4}$. In the case of $\varepsilon_{e, \mu(\tau)}^{u d} \neq 0$, the measured $\theta_{13}$ is no longer robust under NSI [1].

\footnotetext{
${ }^{3}$ This is due to the $V-A$ structure in Eq. (1) [3].

${ }^{4} \mathrm{Also}$, there is large impact of the new phases on the bounds.
} 


\section{NC-like NSI signatures}

The sensitivity to the NC-like NSI is driven by the neutrino-matter interactions. Therefore, long baseline neutrino (LBL) experiments like NOvA and the future DUNE are sensitive to $\varepsilon_{\alpha \beta}^{m}$ NSI couplings. From a global fit, using only neutrino oscillation data, constraints on the $\varepsilon_{\alpha \beta}-\left.\varepsilon_{\mu \mu}\right|^{f=d(u) 5}$ for the LMA solution [5] imply $\left|\varepsilon_{\alpha \beta}^{m}\right| \sim 0.05-1$. In particular, one of the less constrained and non-diagonal NSI coupling is $\varepsilon_{e \tau}^{m}$ which plays an important role in the determination of the CP violation in the lepton sector. Since the CC-like NSI couplings are more constrained than the NC ones, we have neglected them in the analysis of LBL experiments.

A plethora of works dedicated to constrain NC-like NSI couplings in different LBL neutrino oscillation experiments (and from neutrino scattering) can be found in the literature (see Ref. [6] and the references included). In Ref. [5] it has been shown that the measured neutrino oscillation parameters are robust in presence of NSI. However, this is not the case of the Dirac CP phase, which is to be determined. More than extracting bounds we are interested in establishing the the degree of 'confusion' in the determination of the Dirac CP phase in presence of NSI. For simplicity we have considered only the off-diagonal NSI parameter $\varepsilon_{e \tau}^{m} \equiv|\varepsilon| \exp (i \phi) \neq 0$ and in the analysis we considered only the (Anti)neutrino appearance channel. To quantify the effect, we have simulated true neutrino events (including NSI) and test SM events in both T2K (scaled 5 yrs) and NOvA $(3 v+3 \bar{v})$. We found that, thanks to 'parameter degeneracies' if the current prefer value for $\delta_{\mathrm{CP}}^{\text {True }} \sim-\pi / 2$ were established, with current facilities, we can not disentangle whether the origin of the CP violation comes from the usual Dirac CP violating phase or from the NSI couplings (even with $\phi=\pi$ ) [2].

\section{Acknowledgments}

I want to thank my collaborators in Ref. [1, 2] from which this proceeding is based. This work was supported by the U.S. DOE under the DE-SC0013632 and DE-SC0009973 contracts.

\section{References}

[1] S. K. Agarwalla, P. Bagchi, D. V. Forero and M. Tórtola, JHEP 1507 (2015) 060 doi:10.1007/JHEP07(2015)060 [arXiv:1412.1064 [hep-ph]].

[2] D. V. Forero and P. Huber, Phys. Rev. Lett. 117 (2016) no.3, 031801 doi:10.1103/PhysRevLett.117.031801 [arXiv:1601.03736 [hep-ph]].

[3] J. Kopp, M. Lindner, T. Ota and J. Sato, Phys. Rev. D 77 (2008) 013007 doi:10.1103/PhysRevD.77.013007 [arXiv:0708.0152 [hep-ph]].

[4] C. Biggio, M. Blennow and E. Fernandez-Martinez, JHEP 0908 (2009) 090 doi:10.1088/1126-6708/2009/08/090 [arXiv:0907.0097 [hep-ph]].

[5] M. C. Gonzalez-Garcia and M. Maltoni, JHEP 1309 (2013) 152 doi:10.1007/JHEP09(2013)152 [arXiv:1307.3092 [hep-ph]].

[6] O. G. Miranda and H. Nunokawa, New J. Phys. 17 (2015) no.9, 095002 doi:10.1088/1367-2630/17/9/095002 [arXiv:1505.06254 [hep-ph]].

\footnotetext{
${ }^{5}$ For the constrains on $\varepsilon_{\alpha \beta}^{f=e}$ that also come from ' $v-e$ ' scattering see for instance table III in Ref. [6].
} 